\title{
Small-scale gravity waves in ER-2 MMS/MTP wind and temperature measurements during CRYSTAL-FACE
}

\author{
L. Wang ${ }^{1}$, M. J. Alexander ${ }^{1}$, T. P. Bui ${ }^{2}$, and M. J. Mahoney ${ }^{3}$ \\ ${ }^{1}$ NorthWest Research Associates, Inc., Colorado Research Associates Div., 3380 Mitchell Lane, Boulder, CO 80301, USA \\ ${ }^{2}$ NASA Ames Research Center, Moffett, CA 94035, USA \\ ${ }^{3}$ Jet Propulsion Laboratory, California Institute of Technology, Pasadena, CA 91109, USA
}

Received: 10 October 2005 - Published in Atmos. Chem. Phys. Discuss.: 10 November 2005

Revised: 6 February 2006 - Accepted: 8 February 2006 - Published: 3 April 2006

\begin{abstract}
Lower stratospheric wind and temperature measurements made from NASA's high-altitude ER-2 research aircraft during the CRYSTAL-FACE campaign in July 2002 were analyzed to retrieve information on small scale gravity waves (GWs) at the aircraft's flight level (typically $\sim 20 \mathrm{~km}$ altitude). For a given flight segment, the S-transform (a Gaussian wavelet transform) was used to search for and identify small horizontal scale GW events, and to estimate their apparent horizontal wavelengths. The horizontal propagation directions of the events were determined using the Stokes parameter method combined with the cross S-transform analysis. The vertical temperature gradient was used to determine the vertical wavelengths of the events. GW momentum fluxes were calculated from the cross S-transform. Other wave parameters such as intrinsic frequencies $\hat{\omega}$ were calculated using the $\mathrm{GW}$ dispersion relation. More than $100 \mathrm{GW}$ events were identified. They were generally high frequency waves with vertical wavelength of $\sim 5 \mathrm{~km}$ and horizontal wavelength generally shorter than $20 \mathrm{~km}$. Their intrinsic propagation directions were predominantly toward the east, whereas their ground-based propagation directions were primarily toward the west. Among the events, $\sim 20 \%$ of them had very short horizontal wavelength, very high intrinsic frequency, and relatively small momentum fluxes, and thus they were likely trapped in the lower stratosphere.

Using the estimated GW parameters and the background winds and stabilities from the NCAR/NCEP reanalysis data, we were able to trace the sources of the events using a simple reverse ray-tracing. More than $70 \%$ of the events were traced back to convective sources in the troposphere, and the sources were generally located upstream of the locations of the events observed at the aircraft level. Finally, a probability density function of the reversible cooling rate due to
\end{abstract}

Correspondence to: L. Wang

(lwang@cora.nwra.com)
GWs was obtained in this study, which may be useful for cirrus cloud models.

\section{Introduction}

GWs are commonly observed at all levels in the atmosphere. Their role in the global circulation became widely appreciated twenty years ago when their effects were first parameterized in global circulation models (Palmer et al., 1986; McFarlane, 1987). The wave effects on the global circulation are quite sensitive to the properties of waves, e.g., their phase speeds, horizontal and vertical wavelengths, and the momentum flux they carry (e.g. Holton, 1982; Alexander and Dunkerton, 1999). Hence, numerous observational studies have attempted to quantify these properties (see Fritts and Alexander, 2003). Most data sets do not provide enough information to quantify all the needed wave properties (Bacmeister et al., 1990a, b, 1996; Pfister et al., 1993; Alexander and Pfister, 1995; Alexander et al., 2000), but measurements from the ER-2 aircraft platform during the Cirrus Regional Study of Tropical Anvils and Cirrus Layers Florida Area Cirrus Experiment (CRYSTAL-FACE) (Jensen et al., 2004) do. Specifically, simultaneous measurements of 3-dimensional vector winds from the Meteorological Measurement System (MMS), and temperatures and vertical temperature gradients from the Microwave Temperature Profiler (MTP) can be combined to fully characterize the waves sampled by the ER-2 in the lower stratosphere.

Convection is one of the globally important sources of GWs, and the CRYSTAL-FACE flights in the vicinity of subtropical and tropical deep convection provide a wealth of data on GWs from this source. One motivation of this study is to provide observational constraints for parameterizations developed to describe the effects of convectively generated

Published by Copernicus GmbH on behalf of the European Geosciences Union. 


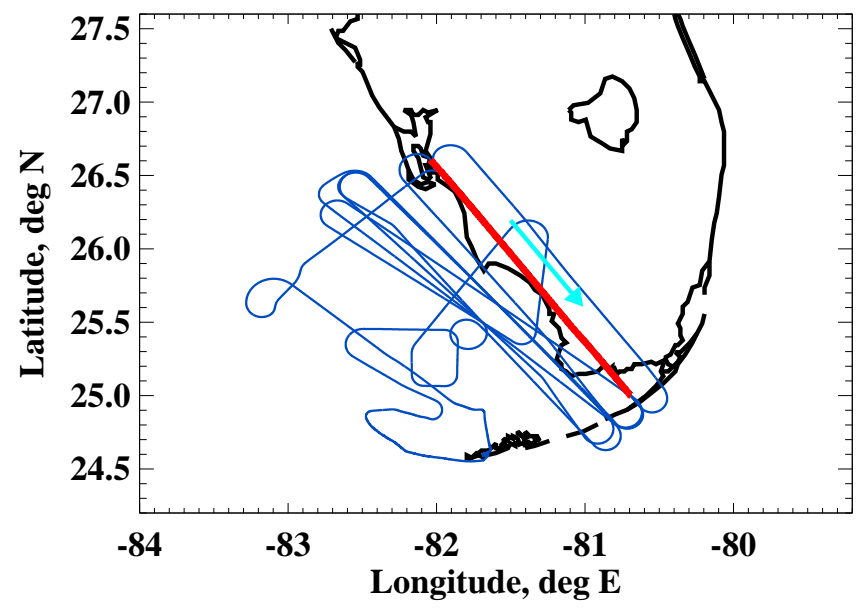

Fig. 1. The ER-2 flight track (blue line) during 7 July 2002 16:0122:39 UTC. The red line shows the flight segment to be analyzed as a case study in this paper. The arrow indicates the direction of the flight segment. In total, there were 10 flights from which 136 such flight segments were identified. See text for further details.

GWs (Chun and Baik, 1998, 2002; Beres et al., 2004), whose simplifying assumptions are poorly validated to date.

Recent studies have shown that GWs in the upper troposphere and lower stratosphere influence the formation of high cirrus clouds considerably (Jensen et al., 2001, 2005; Jensen and Pfister, 2004; Haag and Kärcher, 2004) through wave induced temperature oscillations. Cirrus clouds, especially those in the tropical tropopause layer (TTL), play important roles in the Earth's radiation budget and the stratospheric water vapor budget as cirrus formed in situ within the TTL can effectively dehydrate air entering the stratosphere (Jensen et al., 2001; Jensen and Pfister, 2004). Tropical cirrus clouds also influence the stratospheric chemistry indirectly by affecting the stratospheric humidity (Solomon, 1999). One of the main challenges in modeling the effects of cirrus clouds on stratospheric water vapor concentrations, however, is to specify the temperature in the TTL, which is modulated considerably by GWs (Jensen and Pfister, 2004). The focus of this study is to analyze in detail the properties of short horizontal scale GWs in the south Florida region from the MMS and MTP wind and temperature measurements obtained on board the ER-2 aircraft.

The paper is organized as follows. Section 2 describes the wind and temperature data used in this study. Section 3 describes the procedures to identify GW events and to estimate GW parameters such as wavelength, propagation direction, and vertical flux of horizontal momentum of the events. It also shows the statistics of the parameters derived. Section 4 investigates the sources of the wave events. Discussions are given in Sect. 5. In Sect. 6, conclusions are given.

\section{Data}

During the CRYSTAL-FACE campaign in July 2002, multiple sorties of NASA's ER-2 aircraft were carried out over the southern Florida and Caribbean region (12.4-29.9 $\mathrm{N}$, $\left.273.1-283.0^{\circ} \mathrm{E}\right)$ to measure atmospheric properties in the lower stratosphere and upper troposphere. The aircraft flew at $\sim 20 \mathrm{~km}$ altitude and at a cruise speed of $\sim 200 \mathrm{~ms}^{-1}$. The dates and times of all 10 ER-2 flights are listed in Table 1. Each flight started either late in the morning or early in the afternoon, and ended late in the afternoon. Each flight mission lasted $\sim 6-7 \mathrm{~h}$, and covered a total horizontal distance of $\sim 4000-5000 \mathrm{~km}$. Figure 1 shows, for example, the ER-2 flight track during 7 July 2002 between 16:01 and 22:39 UTC.

Among the instruments aboard the ER2 aircraft, the MMS measured in situ winds, temperature, and pressure (Scott et al., 1990), whereas the MTP measured vertical temperature profiles along the flight path by microwave remote sensing (Denning et al., 1989). The precisions of the MMS winds, temperature, and pressure were $0.1 \mathrm{~ms}^{-1}, 0.1 \mathrm{~K}$, and $0.1 \mathrm{mb}$, respectively. The precision of the MTP temperature was $\sim 0.25 \mathrm{~K}$ at the flight level. The original sampling rate of the MMS was $\sim 10 \mathrm{~Hz}$, but the $1 \mathrm{~Hz}$ version was used in this study as it had a better signal-to-noise ratio. Given the ER-2 speed at $\sim 200 \mathrm{~ms}^{-1}$, the horizontal resolution of the MMS measurements was therefore $0.2 \mathrm{~km}$. The sampling rate of the MTP was $0.1 \mathrm{~Hz}$. The along-track horizontal resolution of the MTP temperature was $\sim 2 \mathrm{~km}$. The vertical profile of the MTP temperature generally extended $\sim 9 \mathrm{~km}$ above and below the flight altitude for the measurements analyzed in this study. The vertical resolution of the MTP temperature profile varies with the distance from the aircraft, being $\sim 160 \mathrm{~m}$ at the flight level and $\sim 3 \mathrm{~km}$ at $5 \mathrm{~km}$ from the flight level.

To facilitate GW analyses, we first divided each flight into flight segments (or legs) within which the flight altitude was nearly constant at $\sim 20 \mathrm{~km}$ and the flight path was nearly straight so as to avoid turns and rapid ascents and descents of the aircraft. Also, we required that the length of each segment be no shorter than $50 \mathrm{~km}$. The highlighted portion of the flight track in Fig. 1 shows an example of such a flight segment. In total, 136 flight segments of this kind were selected, ranging from $\sim 50$ to $\sim 1100 \mathrm{~km}$ in length, with the majority in the $\sim 200 \mathrm{~km}$ range. The flights on 9 and 26 July were exceptional in that they contained a few very long flight segments $(\sim 1000 \mathrm{~km}$ or longer) which reached as far south as $\sim 12.4^{\circ} \mathrm{N}$ and deep into the Caribbean. The orientations of the flight segments were somewhat anisotropic in the sense that more segments were aligned zonally than meridionally.

Figure 2 shows the MMS zonal velocities $u$, meridional velocities $v$, vertical velocities $w$, and temperature $T$ for the flight segment highlighted in Fig. 1. Overplotted on the MMS temperature is the MTP temperature at the flight altitude. The raw data were interpolated to a regular 0.2 and 
Table 1. The dates, and times (UTC) of all 10 ER-2 flights during the CRYSTAL-FACE campaign.

\begin{tabular}{lllllllllll}
\hline Date & 3 July & 7 July & 9 July & 11 July & 13 July & 16 July & 19 July & 23 July & 26 July & 28 July \\
\hline Take-off & $14: 36$ & $16: 01$ & $15: 18$ & $14: 59$ & $17: 00$ & $18: 06$ & $17: 02$ & $17: 13$ & $15: 51$ & $16: 56$ \\
Landing & $20: 23$ & $22: 39$ & $21: 11$ & $21: 18$ & $23: 09$ & $23: 59$ & $23: 34$ & $24: 18$ & $21: 14$ & $23: 11$ \\
\hline
\end{tabular}
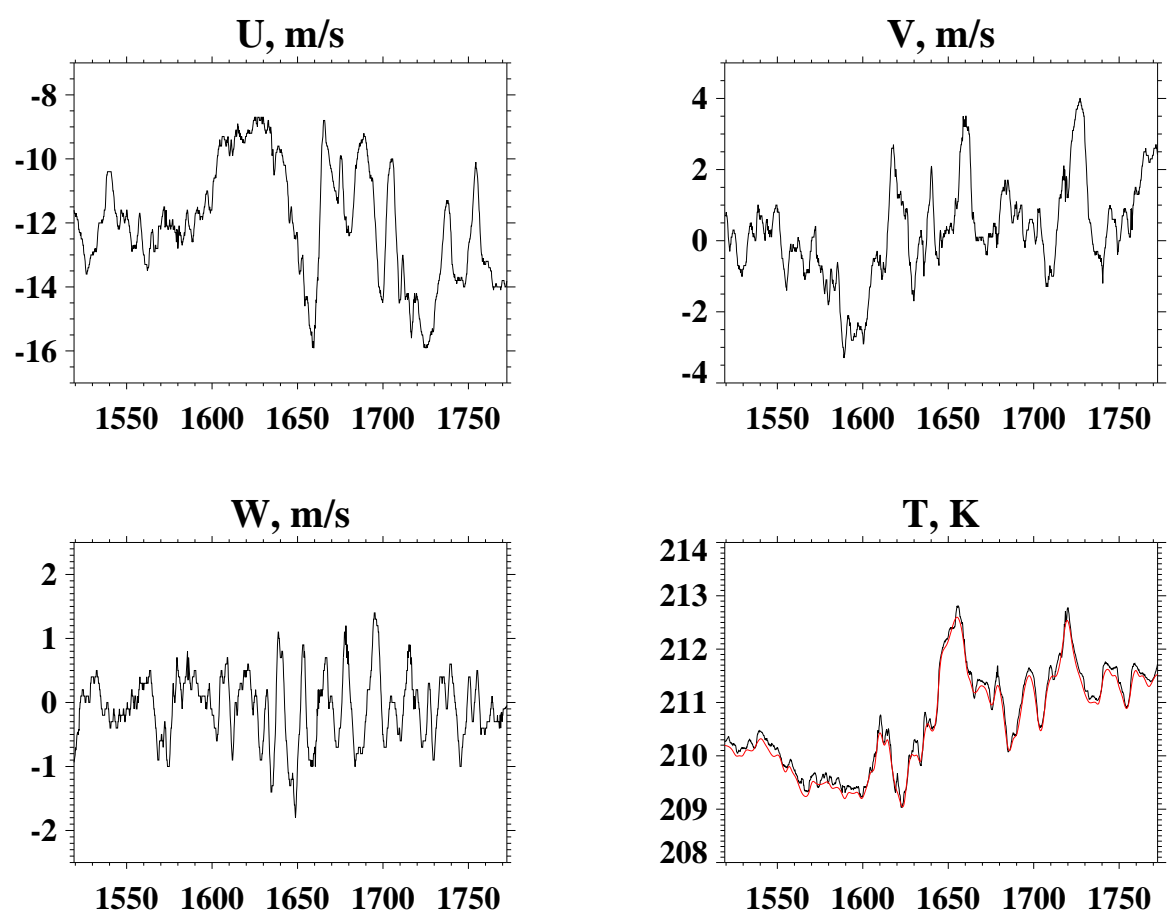

\section{Flight Distance, km}

Fig. 2. MMS winds and temperatures (dark lines) as functions of flight distance for the flight segment highlighted in red in Fig. 1. Also plotted is the MTP temperature at the flight altitude (red line). In this plot, the raw data were interpolated to a regular 0.2 and $2 \mathrm{~km}$ horizontal resolution along the flight track for the MMS and MTP data, respectively.

$2 \mathrm{~km}$ horizontal resolution along the flight track for MMS and MTP data, respectively. The MMS and MTP temperatures generally agreed very well, though the former had a better temporal resolution. Note that localized wavy structures are clearly seen in Fig. 2, especially for horizontal winds and temperatures. It is assumed in this study that these wavy structures are GW events (or packets).

In the next section, we describe the procedure to identify GW events from the flight segments in a more quantitative way, and estimate GW parameters including wavelengths, horizontal propagation directions, and vertical fluxes of horizontal momentum for such events.

\section{GW parameters}

\subsection{GW event and apparent horizontal wavelength}

As mentioned in the previous section, GW packets were clearly seen in the horizontal wind and temperature data for the flight segment shown in Fig. 1. To determine the locations and amplitudes of these wave packets quantitatively, we applied the S-transform to the MMS wind and temperature along the flight segment. The S-transform (Stockwell et al., 1996) is a continuous wavelet transform (CWT) whose basis functions are formed as the product of a Gaussian envelope and sine/cosine functions. The CWT $\Psi_{u}\left(\xi, \lambda_{h}^{\prime}\right)$ of a function, e.g., $u(x)$ is defined by

$\Psi_{u}\left(\xi, \lambda_{h}^{\prime}\right)=\int_{-\infty}^{\infty} u(x) \psi\left(x-\xi, \lambda_{h}^{\prime}\right) d x$

where $x$ and $\xi$ are the horizontal distance in the flight segment, $\lambda_{h}^{\prime}$ is the apparent horizontal wavelength. $\lambda_{h}^{\prime}$ is related 

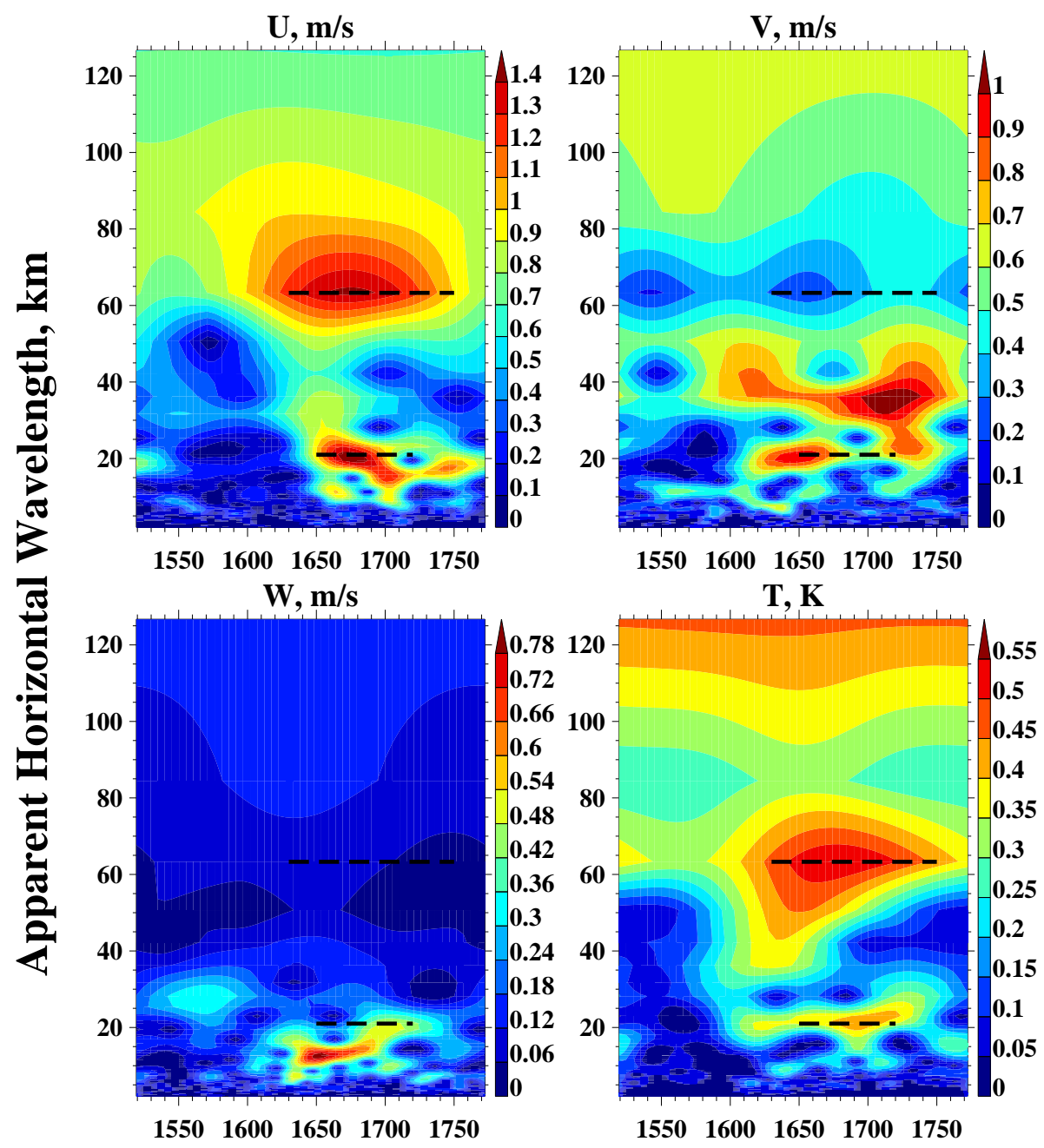

\section{Flight Distance, km}

Fig. 3. Contours of horizontal distance vs. apparent horizontal wavelength $\lambda_{h}^{\prime}$ of S-transform amplitudes for the regularly interpolated MMS winds and temperatures shown in Fig. 2. The contour interval is $0.1 \mathrm{~ms}^{-1}$ for zonal and meridional winds, $0.06 \mathrm{~ms}^{-1}$ for vertical wind, and $0.05 \mathrm{~K}$ for temperature. The dashed lines show the horizontal spans of the GW events identified.

to the true GW horizontal wavelength $\lambda_{h}$ by $\lambda_{h}=\lambda_{h}^{\prime}|\cos \theta|$, where $\theta$ is the angle between the flight direction $\gamma$ and the GW horizontal propagation direction $\phi$, which will be discussed in the next subsection. The mother wavelet $\psi$ is defined as

$$
\psi\left(x, k^{\prime}\right)=\frac{\left|k^{\prime}\right|}{\sqrt{2 \pi}} e^{-\frac{x^{2} k^{\prime 2}}{2}} e^{-i 2 \pi k^{\prime} x}
$$

where $k^{\prime}$ is the inverse of $\lambda_{h}^{\prime}$, i.e., the apparent horizontal wavenumber. The S-transform method has already been applied to geophysical data and has proved to be useful in estimating wave perturbation amplitudes and phase information (e.g., Stockwell and Lowe, 2001; Wang et al., 2006).

Figure 3 shows contours of horizontal distance vs. apparent horizontal wavelength $\lambda_{h}^{\prime}$ of wave amplitudes in winds and temperatures from the S-transform for the regularly interpolated MMS data shown in Fig. 2. The locations and amplitudes of GW packets are clearly seen in Fig. 3. Notably, there was a strong wave signal with a dominant $\lambda_{h}^{\prime}$ of $\sim 63 \mathrm{~km}$ for both temperature and zonal wind between 1630 and $1750 \mathrm{~km}$ flight distance. The S-transform detected another wave packet with a shorter $\lambda_{h}^{\prime}(\sim 21 \mathrm{~km})$, occurring between 1650 and $1720 \mathrm{~km}$ flight distance. It showed up in all the variables, although less pronounced in $w$ in comparison to the others. In this study, we defined a GW event when strong wave perturbations showed up in both temperature and at least one component of horizontal winds at the same location and at the same $\lambda_{h}^{\prime}$. To achieve the statistical robustness, a statistical testing of the S-transform based on Stockwell (1999) was performed to ensure that the relevant amplitudes 


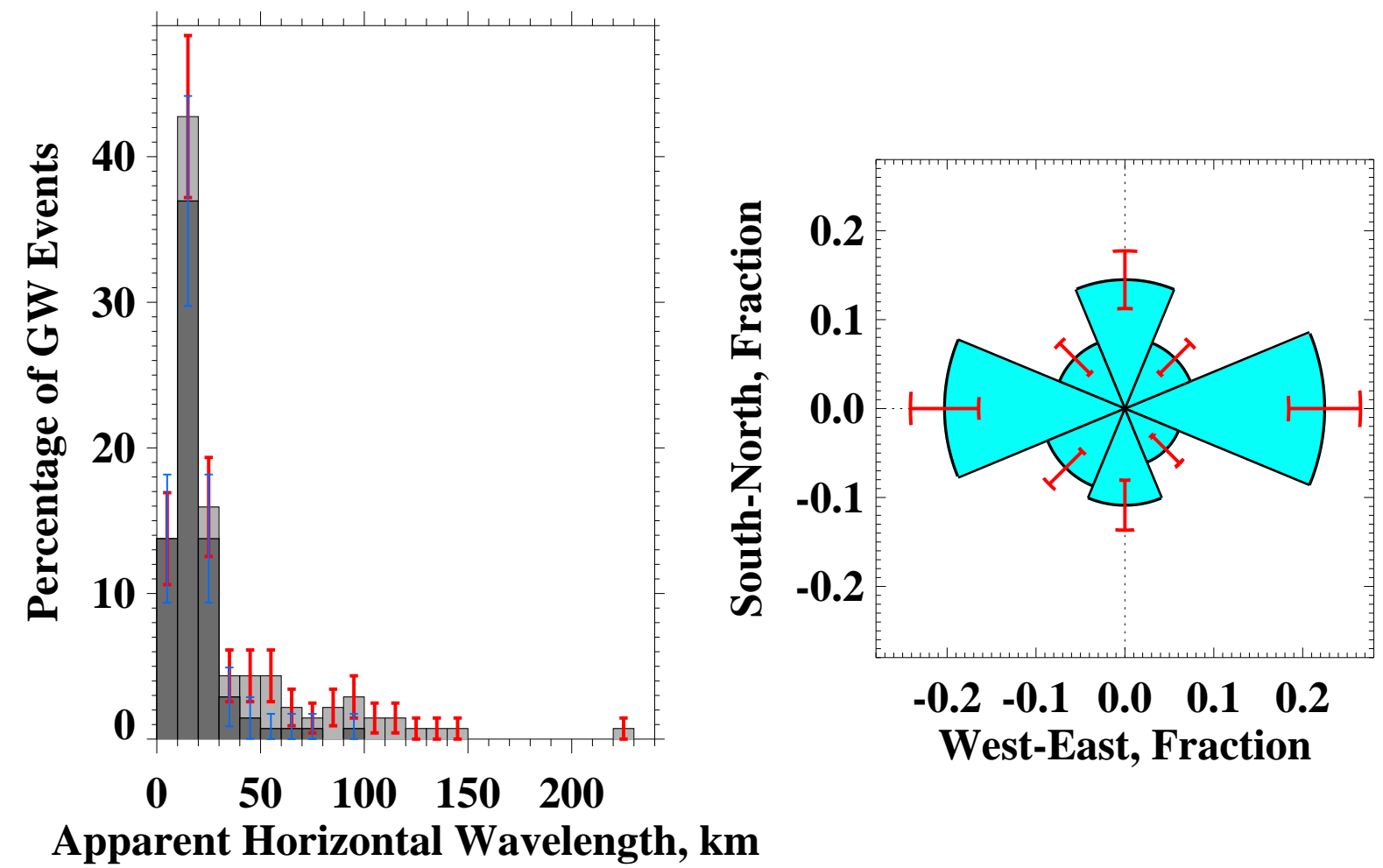

Fig. 4. Histogram (in percentage) of apparent horizontal wavelengths $\lambda_{h}^{\prime}$ for the $138 \mathrm{GW}$ events identified (left panel) and angular distribution of flight directions of the events (right panel). The red error bars indicate the uncertainties of $\lambda_{h}^{\prime}$ based on the Poisson counting statistics. The darker histogram in the left panel includes only those events which had large wave amplitudes in both $w$ and $T$. The corresponding error bars are shown in blue. The error bars of flight directions are shown in red.

were above the $95 \%$ confidence level for each event. Also, we only focused on signals with dominant $\lambda_{h}^{\prime}$ no shorter than $5 \mathrm{~km}$ because very short GWs are more severely affected by processes such as dissipation, reflection, etc., than longer ones, so they are more difficult to ray-trace (e.g., Marks and Eckermann, 1995). In Fig. 3, the dashed lines indicate the horizontal spans of the GW events thus identified for this particular flight segment.

In total, 138 such wave events were identified from the 136 flight segments available. Figure 4 shows the histogram of $\lambda_{h}^{\prime}$ and the angular distribution of the flight directions of the events. Most of the events had a dominant $\lambda_{h}^{\prime}$ of $10-$ $20 \mathrm{~km}$. Also, the average wave amplitudes were $\sim 0.69 \mathrm{~ms}^{-1}$, $0.75 \mathrm{~ms}^{-1}, 0.35 \mathrm{~ms}^{-1}$, and $0.39 \mathrm{~K}$, for $u, v, w$, and $T$, respectively (not shown). Note that such wave amplitudes for $u, v$ and $T$ are much smaller than what are usually seen in inertia GWs typically resolved in radiosonde profiles (e.g., Wang and Geller, 2003). Such smaller values are consistent, via GW polarization relations, with the much higher intrinsic frequencies inferred for these GWs (which will be discussed in the next subsection). Also, note that the ER-2 aircraft flight paths were chosen to avoid areas directly above deep rain events where the most vigorous GW motions might be present. Such a sampling bias in the aircraft data likely causes an underestimation of the actual GW amplitudes in the region. It is worth noting that significant wave perturbations showed up in both MMS $T$ and $w$ for $72 \%$ of the GW events. Statistically, the correspondence between MMS $w$ and $T$ was best for GW events with short $\lambda_{h}^{\prime}$, and it worsened as $\lambda_{h}^{\prime}$ increased. Such a correspondence held up for $\lambda_{h}^{\prime}$ as long as $\sim 90 \mathrm{~km}$ (Fig. 4).

\subsection{Vertical wavelength and horizontal propagation direc-} tion

The dominant vertical wavelengths $\lambda_{z}$ of the GW events identified above were estimated using the MTP vertical temperature gradient as follows:

$\lambda_{z}=2 \pi\left|\frac{i T^{\prime}}{d T^{\prime} / d z}\right| \sim 2 \pi \frac{\sigma\left(T^{\prime}\right)}{\sigma\left(d T^{\prime} / d z\right)}$

where $T^{\prime}$ is the GW perturbation from the MTP temperature, and $\sigma\left(T^{\prime}\right)$ is the standard deviation of $T^{\prime}$, i.e.,

$\sigma\left(T^{\prime}\right)=\sqrt{\frac{1}{n-1} \sum_{i=1}^{n}\left(T_{i}^{\prime}-\overline{T^{\prime}}\right)^{2}}$

We used the standard deviation because single realizations of ratio $T^{\prime} /\left(d T^{\prime} / d z\right)$ can be singular while the ratio of the 
standard deviations gives the correct result for a theoretical monochromatic wave. $T^{\prime}$ was derived by linearly detrending the MTP temperature for each event. To increase the statistical confidence of the standard deviations in Eq. (3), in practice, we calculated $\lambda_{z}$ for only those events which had at least 6 MTP temperature measurements available. For the two GW events identified in Fig. $3, \lambda_{z}$ was 7.2 and $8.1 \mathrm{~km}$ for the ones with longer and shorter $\lambda_{h}^{\prime}$, respectively. Note that three of the events (two on 3 July and one on 13 July) had too few MTP measurements to determine their $\lambda_{z}$.

According to linear GW theory, the (intrinsic) horizontal propagation direction of a $\mathrm{GW} \phi$ is aligned with the major axis of the wind perturbation hodograph (Gossard and Hooke, 1975). Such a property of GWs has been used in previous studies to estimate $\phi$ from vertical profiles of winds and temperatures with the aid of various techniques including the Stokes parameter method, the hodographic analysis, among others (see Eckermann, 1996, for a review of the different techniques). As noted by Eckermann (1996), those methods are inherently related and mathematically equivalent. We derived $\phi$ from GW wind and temperature perturbations along flight track using a variant of the Stokes-parameter technique (Vincent and Fritts, 1987; Eckermann and Vincent, 1989). Briefly, for a monochromatic GW, the relevant Stokes parameters are defined as

$D=\tilde{u}^{2}-\tilde{v}^{2}$

$P=2 \tilde{u} \tilde{v} \cos \delta$

where $\tilde{u}$ and $\tilde{v}$ are the amplitudes of $u$ and $v$, respectively, and $\delta$ is $\left(\Phi_{v}-\Phi_{u}\right)$, i.e., the phase difference between $v$ and $u$. In optical terms, $D$ is the throughput anisotropy parameter, and $P$ is the linear polarization parameter. The major axis orientation $\phi^{\prime}$ of GW wind perturbation hodograph is given by (Kraus, 1966)

$\phi^{\prime}=\frac{1}{2} \arctan \left(\frac{P}{D}\right)$

In practice, $\tilde{u}$ and $\tilde{v}$ were calculated directly from the Stransform amplitudes of the MMS winds for the GW events identified. The phase difference $\delta$ was calculated by performing a cross-S-transform analysis (cross ST) of the MMS winds in analogy with cross-spectral analysis using the Fourier transform. The cross ST of two time series $h(x)$ and $g(x)$ is defined as

$W_{h}(\xi, k)\left\{W_{g}(\xi, k)\right\}^{*}$

where $\left\{W_{g}(\xi, k)\right\}^{*}$ is the complex conjugate of $W_{g}(\xi, k)$. The phase of the cross ST can be shown to be equivalent to the phase difference between $g(x)$ and $h(x)$, $\Phi(\xi, k)_{g}-\Phi(\xi, k)_{h}$.

There is a $180^{\circ}$ ambiguity of $\phi^{\prime}$, however, since $\phi^{\prime}$ can only vary between $[-\pi / 2, \pi / 2]$. To solve such an ambiguity, we used the additional information of temperature. It can be shown that the phase difference between $T$ and $u$ along the flight track is (see Appendix A)

$\Phi_{T}-\Phi_{u}=\arctan \left(\frac{\tilde{v} \sin (\phi) \sin (\delta)}{\tilde{u} \cos (\phi)+\tilde{v} \sin (\phi) \cos (\delta)}\right)-\frac{\pi}{2}$

where again, in practice, $\left(\Phi_{T}-\Phi_{u}\right)$ was calculated using the cross ST. $\phi$ is either $\phi^{\prime}$ or $\left(\phi^{\prime}+\pi\right)$ depending on which one satisfies Eq. (9) more closely. Note that very high intrinsic frequency GWs are more linearly polarized, thus their $\delta$ are close to 0 or $\pi$. Eq. (9) is then reduced to

$\Phi_{T}-\Phi_{u}= \pm \frac{\pi}{2}$

$\phi$ is either $\phi^{\prime}$ or $\left(\phi^{\prime}+\pi\right)$ depending on the phase difference between $T$ and $u$. Such a simplified relation is consistent with Eq. (A10) in the appendix, which is equivalent to the $\mathrm{GW}$ polarization relation for a linearly polarized GW.

With $\phi$ derived, the true dominant horizontal wavelength $\lambda_{h}$ is simply $\phi^{\prime}|\cos \theta|=\phi^{\prime}|\cos (\phi-\gamma)|$, as mentioned in the previous subsection. As expected, $\lambda_{h}$ was shorter than $\lambda_{h}^{\prime}$, and most of the events had $\lambda_{h}$ between 5 and $15 \mathrm{~km}$ (not shown). In comparison, $\lambda_{h}^{\prime}$ was mostly $10-20 \mathrm{~km}$. Other wave parameters such as intrinsic frequency $\hat{\omega}$, group velocity, and intrinsic horizontal phase speed, were determined from the GW dispersion relation

$\frac{1}{\lambda_{z}{ }^{2}}=\frac{1}{\lambda_{h}{ }^{2}} \frac{\left(N^{2}-\hat{\omega}^{2}\right)}{\left(\hat{\omega}^{2}-f^{2}\right)}-\frac{1}{4 H_{\rho}{ }^{2}}$

where $H_{\rho}$ is the density scale height calculated from the MTP data. For the two GW events identified in Fig. $3, \phi, \lambda_{h}$, and $\hat{\omega}$ were $\sim 352^{\circ}, 44.6 \mathrm{~km}$, and $51 f(=0.0032$ radians per second, $f$ is the Coriolis parameter), respectively, for the event with longer $\lambda_{h}^{\prime}$, and $\sim 154^{\circ}, 18.7 \mathrm{~km}$, and $126 f(=0.0079 \mathrm{ra}-$ dians per second), respectively, for the event with shorter $\lambda_{h}^{\prime}$ ( $\phi$ is measured counter clockwise from the East). For the two events, the ratios of Brunt-V $\ddot{a}$ is $\ddot{a} l \ddot{a}$ frequency $N$ derived from the MTP data and $f$ were $\sim 360$. Note that $\hat{\omega}$ can also be derived from the Stokes parameter method since $\hat{\omega} / f$ is equal to the GW perturbation ellipse axial ratio which can be related to some Stokes parameters (e.g., Vincent and Fritts, 1987; Eckermann and Vincent, 1989). We chose not to use this approach since we were mostly dealing with short horizontal scale and high intrinsic frequency waves which are more linearly polarized and thus they cannot be diagnosed for their ellipticities to infer intrinsic frequencies. Furthermore, as mentioned before, more linearly polarized GWs have $\delta$ close to 0 or $\pi$. Indeed, we found that the values of $\delta$ generally cluster around 0 or $\pi$ (not shown), being consistent with these waves having high $\hat{\omega}$.

Figure 5 shows the histogram of $\lambda_{z}$ and the angular distribution of $\phi$ for the GW events. The waves generally had $\lambda_{z}$ of $\sim 5 \mathrm{~km}$ and they propagated predominantly eastward. There also appeared to be a northward bias in $\phi$. The waves were generally short horizontal scale and high intrinsic frequency 


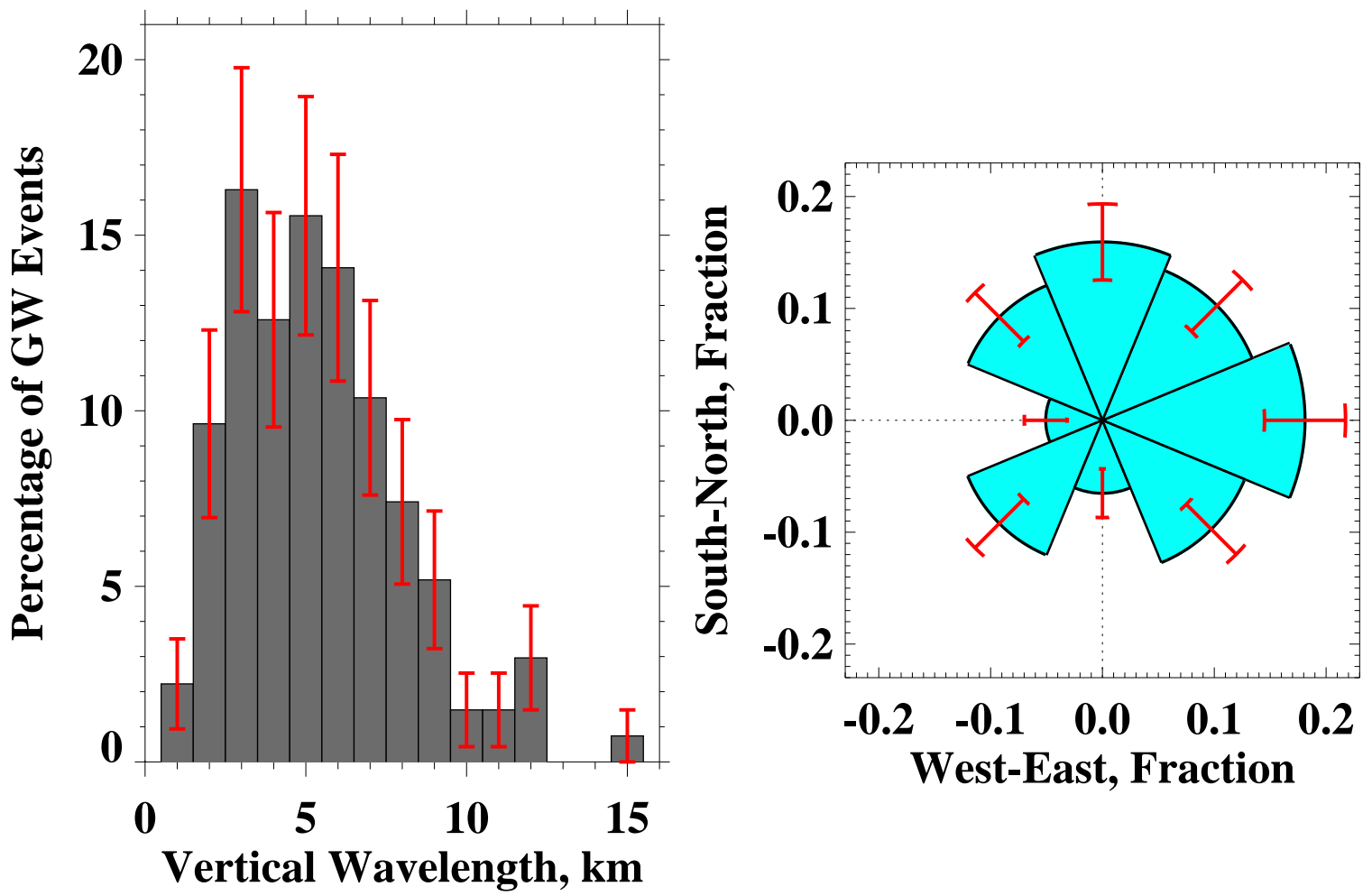

Fig. 5. Histogram (in percentage) of vertical wavelengths $\lambda_{z}$, and the angular distribution of horizontal propagation directions $\phi$ for the GW events identified. Note that 138 events were included in the plot for $\phi$, whereas 135 were included for $\lambda_{z}$. The red error bars are based on the Poisson counting statistics. See text for further details.

with $\lambda_{h}$ generally shorter than $20 \mathrm{~km}$ and $\hat{\omega}$ higher than $13 f$ (not shown). The averaged horizontal intrinsic phase speed and magnitude of horizontal ground-based group velocity were $\sim 13$ and $18 \mathrm{~ms}^{-1}$, respectively (not shown). Finally, note that since $\lambda_{z}$ was undetermined for three of the events, $\hat{\omega} / f$, intrinsic phase speed and ground-based group velocity were also undetermined for them.

\subsection{Momentum flux}

Assuming a locally horizontally homogeneous atmosphere, the GW induced background wind acceleration $(\bar{X}, \bar{Y})$ is related to the vertical gradient of the vertical flux of horizontal momentum $\left(F_{p x}, F_{p y}\right)$ (or simply put, momentum flux) by

$$
(\bar{X}, \bar{Y})=-\frac{\epsilon}{\bar{\rho}} \frac{\partial}{\partial z}\left(F_{p x}, F_{p y}\right)
$$

where $\epsilon$ is an intermittency factor (Alexander and Dunkerton, $1999), \bar{\rho}$ is the background density, and $\left(F_{p x}, F_{p y}\right)$ is defined as

$$
\left(F_{p x}, F_{p y}\right)=\bar{\rho}\left[\left(1-f^{2} / \hat{\omega}^{2}\right)\left(\overline{u^{\prime} w^{\prime}}, \overline{v^{\prime} w^{\prime}}\right)\right]
$$

Hence, momentum fluxes are very important quantities which are related directly to the effects of GWs on the background atmosphere. With MMS vertical velocity available, we were able to calculate the fluxes directly. We estimated momentum fluxes for the GW events using the S-transform and cross ST analysis. For a monochromatic GW, Eq. (13) leads to

$$
\begin{aligned}
& F_{p x}=\frac{1}{2} \bar{\rho}\left(1-f^{2} / \hat{\omega}^{2}\right) \tilde{u} \tilde{w} \cos \left(\Phi_{w}-\Phi_{u}\right) \\
& F_{p y}=\frac{1}{2} \bar{\rho}\left(1-f^{2} / \hat{\omega}^{2}\right) \tilde{v} \tilde{w} \cos \left(\Phi_{w}-\Phi_{v}\right)
\end{aligned}
$$

In practice, $\tilde{u} \tilde{w}, \tilde{v} \tilde{w},\left(\Phi_{w}-\Phi_{u}\right)$, and $\left(\Phi_{w}-\Phi_{v}\right)$ were calculated using the cross ST for each event. $\bar{\rho}$ was calculated from the event mean density which was derived from MMS $T$ and pressure. $\hat{\omega}$ was already estimated from the GW dispersion relation as described in the previous subsection. Since a GW event was generally not purely monochromatic, the contributions from horizontal wavelengths adjacent to the dominant horizontal wavelength $\lambda_{h}^{\prime}$ were added to the value at $\lambda_{h}^{\prime}$ to produce the total momentum flux of the wave event. For the two events identified in Fig. 3, $\left(F_{p x}, F_{p y}\right)$ was $\sim(0.008,0)$ and $(-0.06,-0.004) \mathrm{kg} \mathrm{m}^{-1} \mathrm{~s}^{-2}$ for the one with longer $\lambda_{h}^{\prime}$ and the one with shorter $\lambda_{h}^{\prime}$, respectively. Figure 6 shows the histogram of the magnitudes of momentum flux $\sqrt{F_{p x}^{2}+F_{p y}^{2}}$ for the GW events identified in this study. The averaged magnitude was $\sim 0.026 \mathrm{~kg} \mathrm{~m}^{-1} \mathrm{~s}^{-2}$, and 


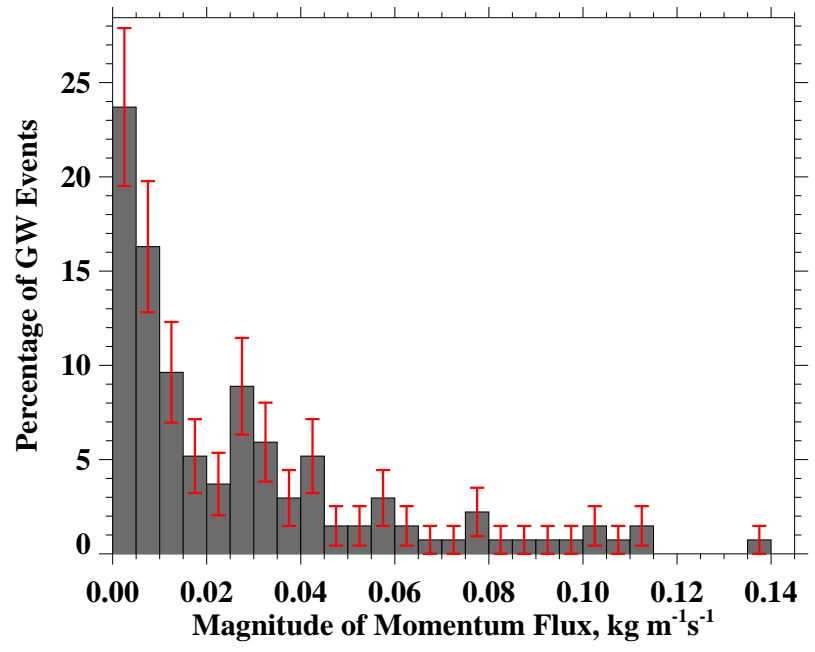

Fig. 6. Histogram (in percentage) of magnitudes of momentum flux $\left(\mathrm{kg} \mathrm{m}^{-1} \mathrm{~s}^{-1}\right)$ for all the 138 events identified. The red error bars are based on the Poisson counting statistics. See text for details.

the maximum magnitude was $\sim 0.13 \mathrm{~kg} \mathrm{~m}^{-1} \mathrm{~s}^{-2}$. These values generally agree with previous estimates of GW momentum fluxes in the lower stratosphere over convection (e.g., Alexander and Pfister, 1995; Alexander et al., 2000).

Note that GWs' horizontal propagation directions can also be derived from the momentum fluxes $\left(F_{p x}, F_{p y}\right)$, as $\tan (\phi)=F_{p y} / F_{p x}$. We compared the propagation directions derived using the flux method with those from the Stokes parameter method, and found that they were similiar for most events. Specifically, their difference was less than $30^{\circ}$ for $\sim 81 \%$ of the events, and the angular distribution of horizontal propagation directions derived using the flux method also showed the eastward bias, as did $\phi$. Large discrepancies existed for $\sim 19 \%$ of the events. We will discuss potential reasons for these discrepancies in Sect. 5.

\section{Wave sources}

To investigate the sources of the GW events, we ray-traced each event back using a simple group velocity method, i.e., $d \boldsymbol{r} / d t=\boldsymbol{C}_{\boldsymbol{g}}$, where $\boldsymbol{r}$ is the position vector of the ray group and $\boldsymbol{C}_{\boldsymbol{g}}$ is the 3-D ground-based group velocity. The initial $\boldsymbol{C}_{\boldsymbol{g}}$ was calculated using the wave parameters derived in the previous section and the background winds and stabilities from the four-times daily NCAR/NCEP reanalysis data. For the subsequent time steps, $\boldsymbol{C}_{\boldsymbol{g}}$ was calculated from the ground-based frequency $\omega$, and horizontal wavenumbers $k, l$ which were assumed to be constant during the ray-tracing. The temporally and spatially varying background $u, v, N$ were estimated from interpolating the reanalysis data to the current time and location of each event. The reverse raytracing was terminated when any of the following conditions was met: the tracing time reached $3 \mathrm{~h}$, the ray reached the

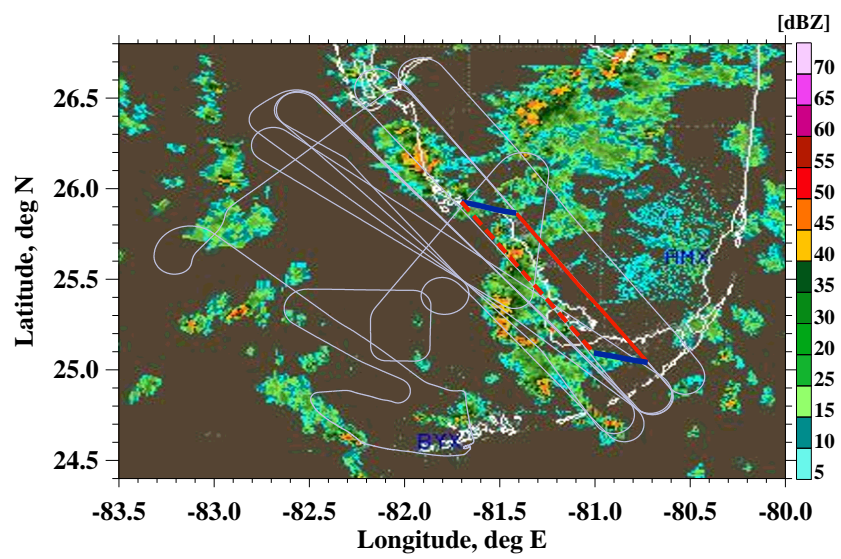

Fig. 7. The NASA LaRC NEXRAD radar base reflectivity composite image on 7 July 2002 17:20 UTC. The thin gray line is the ER-2 flight track on that day. The solid red line denotes the GW event with the longer $\lambda_{h}^{\prime}$ at $\sim 18: 20$ UTC, as shown in Fig. 3 . The blue lines denote the trajectories at both ends of the event reversely ray-traced $1 \mathrm{~h}$ back to 17:20 UTC. The dashed red line connects the end points of the trajectories and indicates where the wave event could have been $1 \mathrm{~h}$ earlier. The blue 3 -character markers are the NEXRAD site codes indicating where the radars are located (AMX: Miami, BYX: Key West). See text for further details.

ground, or the wave was approaching its critical level (where $\hat{\omega} \rightarrow f$ ) or turning point (where $\hat{\omega} \rightarrow N$ ). The horizontal and 3-h-temporal variations of the background fields were generally much smaller in comparison to the vertical variations in the tropical and subtropical region, so the assumption of constant $k$ and $l$ was valid.

The trajectory of each event was compared to the NASA Langley Research Center (LaRC) NEXRAD radar reflectivity images during CRYSTAL-FACE (available every $10 \mathrm{~min})^{1}$ As an example, Fig. 7 shows the NEXRAD radar reflectivity image on 7 July 2002 17:20 UTC. The light gray line is the ER-2 flight track on that day. The solid red line denotes the GW event with the longer $\lambda_{h}^{\prime}$ shown in Fig. 3. The color bar on the side represents base reflectivity in $\mathrm{dBZ}$ and is proportional to precipitation rate. The event was detected at flight level at $\sim 18: 20$ UTC. The solid blue lines denote the trajectories at both ends of the event reversely ray-traced one hour back to 17:20 UTC. The dashed red line connects the end points of the trajectories and indicates where the wave event could have been one hour earlier. The altitude corresponding to the dashed red line was $\sim 7.5 \mathrm{~km}$ in the mid troposphere for both end points. Visual inspection of Fig. 7 shows that the wave event was located over a convectively active region (with base reflectivity larger than $\sim 40 \mathrm{dBz}$ ) in the troposphere one hour before it was observed in the lower stratosphere by the ER-2 aircraft, implying that the source of

\footnotetext{
${ }^{1}$ They can be accessed from the following website: http:// www-angler.larc.nasa.gov/crystal/.
} 

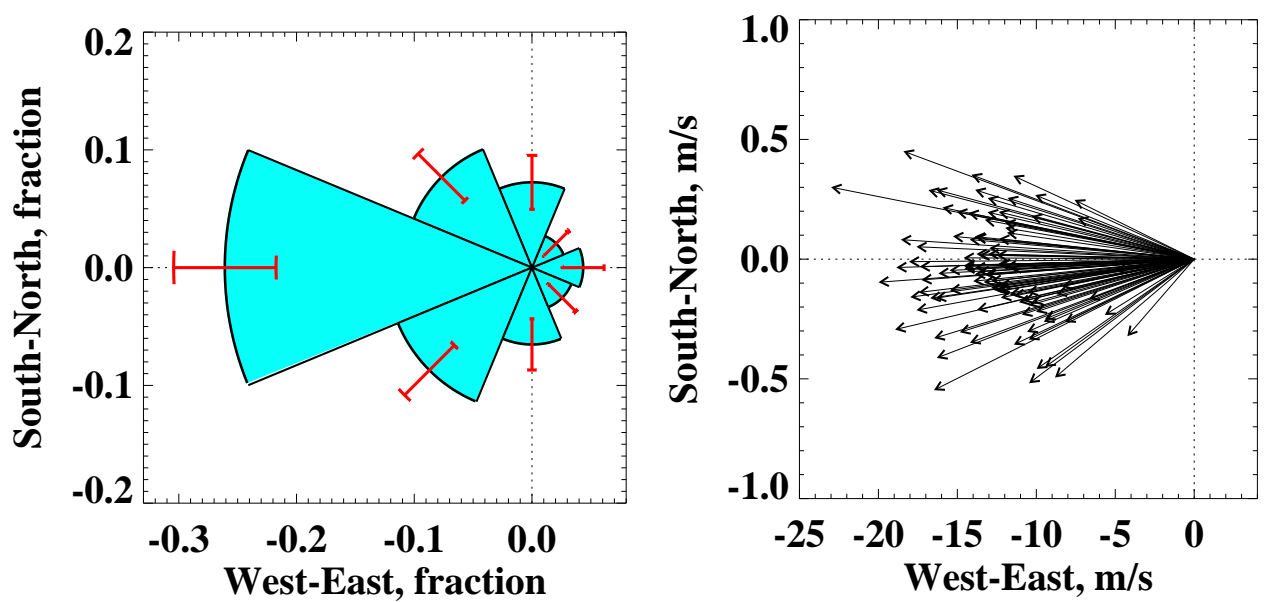

Fig. 8. Angular distribution of source to event directions for those events related to convective sources (102 in total) (left panel), and the scatter plot of their background winds at flight level $(\sim 20 \mathrm{~km})$ (right panel). The red error bars are based on the Poisson counting statistics.

the event was likely the strong convection in the troposphere at $\sim\left(25.5^{\circ} \mathrm{N}, 81.3^{\circ} \mathrm{W}\right)$.

We applied the same procedure to the rest of the events. Of the 135 events for which we conducted ray-tracing (raytracing was not possible for the remaining three events since their $\lambda_{z}$ could not be determined due to the gap in MTP temperature data), $\sim 76 \%$ of them were traced back to convective sources (with base reflectivity larger than $\sim 40 \mathrm{dBz}$ ) below $13 \mathrm{~km}$ in the troposphere (the average tropopause height was $\sim 15 \mathrm{~km}$ ). This is not surprising since convection was expected to be the major source of GWs for these CRYSTALFACE flights. For those GW events which could not be traced back to convective sources in the troposphere, most had shorter $\lambda_{h}$ and higher $\hat{\omega}$ at $20 \mathrm{~km}$, and the ray tracing gave turning points $(\hat{\omega} \rightarrow N)$ somewhere between 13 and $20 \mathrm{~km}$.

Figure 8 shows the angular distribution of source to event directions for those events related to convective sources (102 in total), and the scatter plot of their background winds at flight level. The source to event direction was defined as the direction from the each convective source we identified to the mid-point of the event. Most of the sources were located upstream of the events.

\section{Discussion}

As shown in Fig. 4, coherent wave perturbations showed up in both MMS $T$ and $w$ for $72 \%$ of the GW events, and the coherence was generally better for shorter $\lambda_{h}^{\prime}$ (and $\lambda_{h}$ as well, not shown). The GW polarization relation between vertical velocity and temperature is

$\tilde{w}=\frac{i \hat{\omega} g}{N^{2}} \tilde{T}^{\prime} \sim \frac{i \lambda_{z} g}{N \lambda_{h}} \tilde{T}^{\prime}$ where $g$ is the gravitational acceleration, $\tilde{T}^{\prime}$ is the GW perturbation temperature amplitude divided by the background temperature, and other notations were defined previously. The approximation in Eq. (16) follows from an approximation to the GW dispersion relation when $f \ll \hat{\omega} \ll N$. Evidently, for a given $\tilde{T}^{\prime}$, a GW with longer $\lambda_{h}$ has smaller $\tilde{w}$, and vice versa. Thus, we expect that the coherence between $T$ and $w$ to deteriorate for longer horizontal scale GWs. In general, for longer horizontal scale waves, higher signal to noise ratio for vertical velocity measurements is needed as $\tilde{w}$ gets smaller.

Most of the wave events identified in this study were found to have short horizontal wavelengths and high intrinsic frequencies, as mentioned in Sect. 3. Such waves are prone to be trapped in the atmosphere via high $\hat{\omega}$ reflection (Isler et al., 1997; Swenson et al., 2000; Marks and Eckermann, 1995), and the trapped waves carry no net vertical flux of horizontal momentum. To see how many of the waves events were likely trapped or evanescent waves instead of propagating waves, we show on the left panel of Fig. 9 the percentage of GW events within each $\lambda_{h}$ and $\hat{\omega} / N$ bin. 135 events were included in the plot. Bins are blank if no $\mathrm{GW}$ events fall into them. The right panel of Fig. 9 shows the corresponding binaveraged magnitudes of momentum flux (i.e., $\sqrt{F_{p x}^{2}+F_{p y}^{2}}$ ) of the events. Evidently, most of the events had $\hat{\omega} / N$ equal to or larger than 0.1 and $\lambda_{h}$ shorter than $20 \mathrm{~km}$. In contrast, GWs observed from radiosondes (e.g., Vincent and Alexander, 2000; Wang, 2003; Wang et al., 2005), generally had $\hat{\omega} / N$ less than 0.01 and $\lambda_{h}$ of $\sim 1000 \mathrm{~km}$ or longer at similar latitudes. Generally, shorter horizontal scale GWs had larger momentum fluxes and the largest fluxes occurred for GWs with $\lambda_{h}$ less than $10 \mathrm{~km}$ and $\hat{\omega} / N$ between 0.4 and 0.5 . Interestingly, all the GW events which had $\hat{\omega} / N$ equal to or larger than 0.8 had $\lambda_{h}$ shorter than $10 \mathrm{~km}$, and their momentum fluxes were considerably smaller than those GWs with 


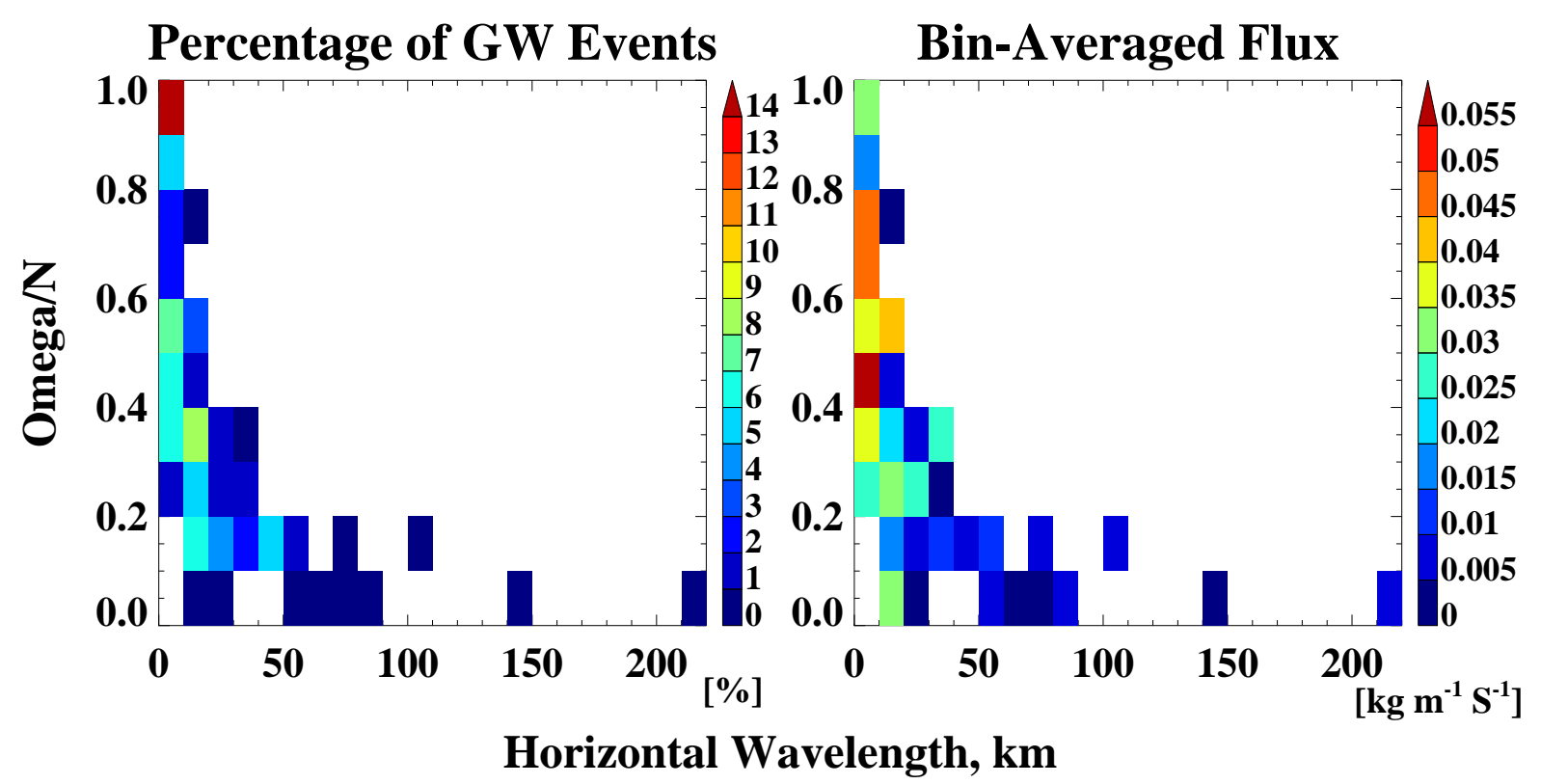

Fig. 9. Percentages of GW events within $\lambda_{h}$ and $\hat{\omega} / N$ bins (left panel), and bin-averaged momentum flux magnitudes (kg m $\left.{ }^{-1} \mathrm{~s}^{-1}\right)($ right panel). See text for further details.

similar $\lambda_{h}$ but relatively lower $\hat{\omega} / N$, thus suggesting that these very high frequency GWs were likely trapped waves. In total, there were 27 such likely trapped events (i.e., $20 \%$ of the 135 events whose $\hat{\omega} / N$ were determined). None of the reverse ray-traces of these very high intrinsic frequency waves could extend down to the troposphere below $13 \mathrm{~km}$ (not shown). The uncertainty in our calculations, however, does not warrant further detailed analysis of wave trapping.

As mentioned in Sect. 3, the intrinsic horizontal propagation directions of the events were predominantly eastward (Fig. 5). Similar anisotropies of GW horizontal propagations have been observed in previous studies in the tropical and subtropical lower stratosphere (e.g., Vincent and Alexander, 2000; Wang, 2003). As mentioned above, our results are distinct in that the GWs examined in this study were short horizontal scale and high intrinsic frequency GWs, whereas the previous radiosonde studies, as referenced here, focused on long horizontal scale and low intrinsic frequency inertio GWs.

Figure 10 shows the typical background wind and temperature from 3-5 times daily high vertical resolution radiosonde observations conducted during the CRYSTALFACE campaign. They were derived from binning the raw balloon data from four south Florida stations (Key West, FL, $24.5^{\circ} \mathrm{N}, 81.8^{\circ} \mathrm{W}$; Miami, FL, 25.8 $\mathrm{N}, 80.4^{\circ} \mathrm{W}$; PARSL mobile facility, $25.8^{\circ} \mathrm{N}, 81.4^{\circ} \mathrm{W}$; and Tampa Bay Area, FL, $27.7^{\circ} \mathrm{N}, 82.4^{\circ} \mathrm{W}$ ) during July 2002 . The error bars indicate the magnitude of the standard deviation for each bin. Zonal winds were generally toward the west. They were very weak in the lower troposphere and increased to $\sim-16 \mathrm{~ms}^{-1}$ at $20 \mathrm{~km}$. Meridional winds were very weak with absolute values less than $4 \mathrm{~ms}^{-1}$ throughout the troposphere and lower stratosphere. The tropopause was well defined and was located at $\sim 15 \mathrm{~km}$.

Note that the prevalent westward background winds offset the eastward anisotropy of intrinsic horizontal propagation directions $\phi$ so that the ground-based propagation directions were actually predominantly westward at slow groundbased phase speeds (not shown). This is consistent with Fig. 9 which showed that that sources were mostly located upstream of the events.

The anisotropy of GW intrinsic horizontal propagation directions (Fig. 5) may be caused largely by the anisotropy of the wave sources, as Alexander and Vincent (2000) and Wang (2003) found for the low intrinsic frequency waves they studied from balloon data, though background wind filtering definitely played a role in modifying the morphology of the waves that we observed. It is difficult to determine the relative importance of the two factors without further analysis and additional data.

As mentioned in the introduction, GWs play a significant role in the dynamics of cirrus cloud formation in the TTL region. One way to quantify their effects in cirrus cloud models is to calculate the the reversible cooling rate that occurs during each wave cycle by $\hat{\omega} \tilde{T}$ (Jensen and Pfister, 2004), where $\tilde{T}$ is the GW temperature perturbation amplitude. Although the waves we observe at ER-2 $20 \mathrm{~km}$ flight altitudes were above the TTL, they must have passed through this region since they were generated by convection below. Direct calculation of $\hat{\omega} \tilde{T}$ has previously only been possible for long-duration Largrangian balloon flights (Hertzog and Vial, 2001), but our analysis is an exception. In fact, $\hat{\omega} \tilde{T}$ is readily 

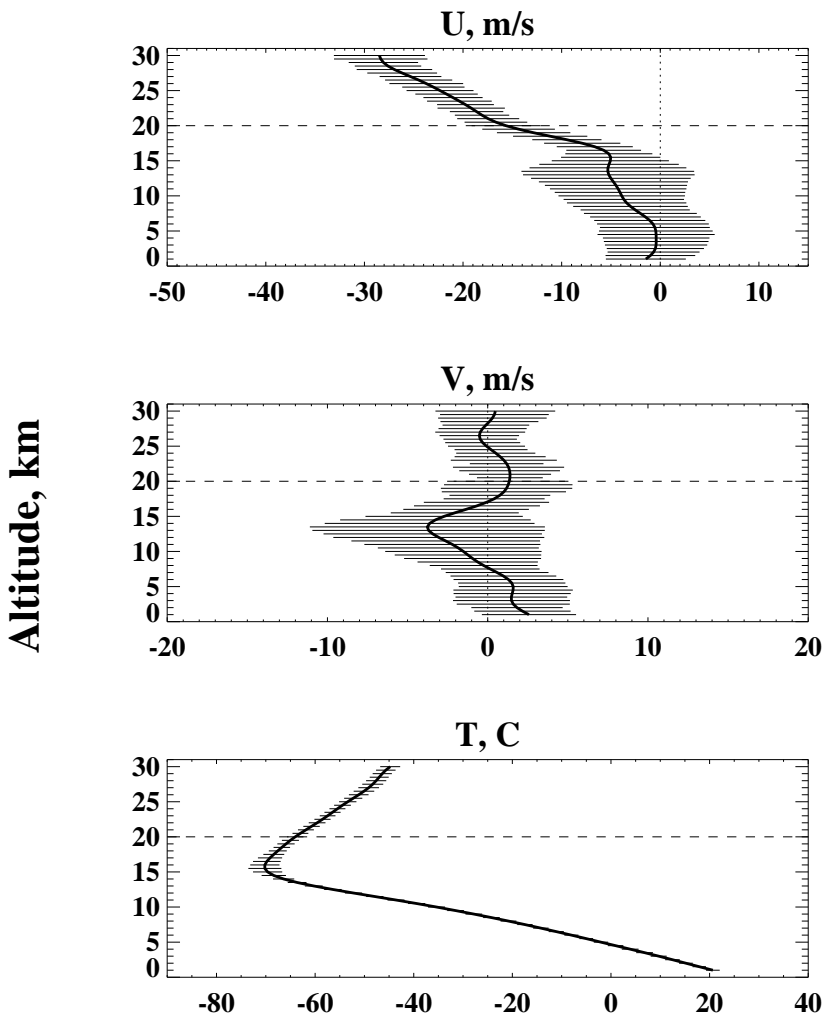

Fig. 10. Typical background winds and temperatures binned from 3-5 times daily high vertical resolution radiosonde observations conducted over the south Florida during the CRYSTAL-FACE campaign. Each error bar is the standard deviation of each bin. The mean flight altitude $(20 \mathrm{~km})$ is marked by a thin dashed line in each plot. See text for further details.

available from this study. Figure 11 shows the probability of observing a GW event with a certain $\hat{\omega} \tilde{T}$. The probability was calculated from the ratio of the sum of the horizontal extents of the GW events (e.g., the lengths of the dashed lines in Fig. 3) having $\hat{\omega} \tilde{T}$ within a certain range and the total distances of all the flight segments examined in this study. Overall, there was a $32 \%$ chance of observing a GW event in ER-2 flights during CRYSTAL-FACE, so the sum of probabilities in Fig. 11 is 0.32 . Most events had $\hat{\omega} \tilde{T}$ less than $22 \mathrm{~K} / \mathrm{h}$

\section{Conclusions}

ER-2 MMS and MTP wind and temperature measurements during CRYSTAL-FACE in July 2002 were analyzed to investigate short horizontal scale GWs at flight altitude $(\sim 20 \mathrm{~km})$. There were $10 \mathrm{ER}-2$ sorties over the southern Florida and the Caribbean region during the campaign (Table 1).

To facilitate GW analysis, we divided each flight into segments (or flight legs) within which the flight altitude was

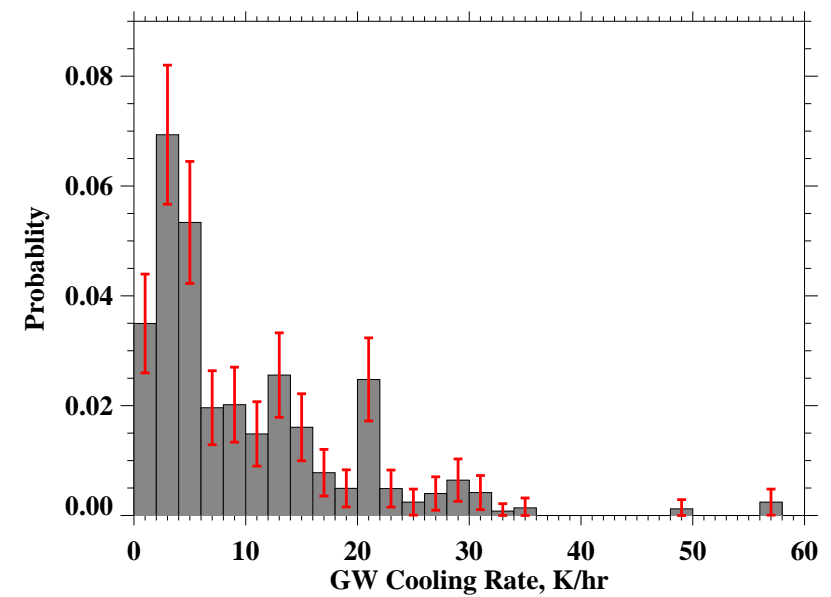

Fig. 11. The probability of observing a GW event with a reversible cooling rate $\hat{\omega} \tilde{T}$ within a given range from the ER-2 aircraft during CRYSTAL-FACE. The red error bars are calculated based on the Poisson counting statistics. See text for further details.

nearly constant at $\sim 20 \mathrm{~km}$ and the flight path was nearly straight so as to avoid turns and rapid ascents and descents of the aircraft. Also, we required that the length of each segment be no shorter than $50 \mathrm{~km}$. 136 such flight segments were selected. We then applied the S-transform (a wavelet transform) to each flight segment to identify GW events in the segment, if there were any. A GW event was identified if coherent wave perturbations showed up in both temperature $T$ and at least one component of horizontal winds at the same location and at the same apparent horizontal wavelength $\lambda_{h}^{\prime}$ (Fig. 3). We only focused on signals with dominant $\lambda_{h}^{\prime}$ no shorter than $5 \mathrm{~km} .138$ such wave events were identified. Meantime, we found that coherent wave perturbations showed up in both $T$ and vertical velocity $w$ for $72 \%$ of the GW events (Fig. 4). The shorter the $\lambda_{h}^{\prime}$ was, the better the correspondence between $w$ and $T$ was, and such a coherence held up for $\lambda_{h}^{\prime}$ up to $\sim 90 \mathrm{~km}$.

The dominant vertical wavelengths $\lambda_{z}$ of the GW events were estimated using both the temperature and vertical temperature gradient oscillations measured along the flight track by MTP. The horizontal propagation directions $\phi$ were estimated from MMS horizontal winds using a variant of the Stokes parameter method with the aid of the cross Stransform. In addition, MMS temperature was used to solve the $180^{\circ}$ ambiguity of $\phi$. The true horizontal wavelengths $\lambda_{h}$ were calculated from $\lambda_{h}^{\prime}$ and the angles between $\phi$ and the flight directions of the events. Other wave parameters such as intrinsic frequencies $\hat{\omega}$, group velocities, and intrinsic horizontal phase speeds, were determined from the GW dispersion relation.

The wave events were found to be generally short horizontal scale and high frequency GWs with $\lambda_{z}$ of $\sim 5 \mathrm{~km}, \lambda_{h}$ generally shorter than $20 \mathrm{~km}$, and $\hat{\omega}$ higher than $13 f$, and they 
propagated their energy and phase predominantly toward the east in the intrinsic frame, i.e., being opposite to the background winds (Fig. 5). The averaged intrinsic phase speed and magnitude of group velocity were $\sim 13$ and $18 \mathrm{~ms}^{-1}$, respectively.

Vertical fluxes of horizontal momentum density of the GW events $\left(F_{p x}, F_{p y}\right)$ were calculated from the MMS winds using the cross $\mathrm{S}$-transform. The averaged momentum flux magnitude was $\sim 0.026 \mathrm{~kg} \mathrm{~m}^{-1} \mathrm{~s}^{-2}$, and the maximum magnitude was $\sim 0.13 \mathrm{~kg} \mathrm{~m}^{-1} \mathrm{~s}^{-2}$ (Fig. 6). We also calculated the horizontal propagation directions of the events using the estimated fluxes and found the results generally agreeing with those from the Stokes parameters method.

We reverse ray-traced the GW events using their 3-D group velocities and the background winds and stabilities from the NCAR/NCEP reanalysis data to locate their sources in the troposphere. The 3-D group velocities were calculated by assuming that the horizontal wavelengths of the events remained constant. The trajectories of the events were compared to the NASA Langley NEXRAD radar reflectivity images available every $10 \mathrm{~min}$ during July 2002 . Of the 135 events for which we were able to perform ray-tracing, $\sim 76 \%$ of them were traced back to convective sources below $13 \mathrm{~km}$ in the troposphere and most of the sources were located upstream of the events (Fig. 8).

Among the 135 events that we were able to determine $\hat{\omega} / N, 20 \%$ of them had very short horizontal wavelength $(<10 \mathrm{~km})$, very high intrinsic frequency $(\hat{\omega} / N \geq 0.8)$, and relatively small momentum fluxes, and thus were likely trapped or evanescent waves.

A probability density function of GW reversible cooling rate was derived from the $\mathrm{GW}$ temperature perturbation amplitudes, intrinsic frequencies, the horizontal extents of the events, and the total distances of flight segments during CRYSTAL-FACE. Such information can be used in cirrus cloud model studies.

\section{Appendix A}

\section{Solving the $180^{\circ}$ ambiguity of GW horizontal propagation direction derived from the Stokes parameters method}

GW perturbation winds and temperature can be written as

$u^{\prime} \equiv \tilde{u} \cos \left(\Phi_{u}\right)$

$v^{\prime} \equiv \tilde{v} \cos \left(\Phi_{v}\right)$

$\hat{T}^{\prime} \equiv \tilde{T} \cos \left(\Phi_{T}\right)$

Let $u_{\|}^{\prime}$ designate the horizontal perturbation velocity parallel to the wave vector (or the propagation direction) and let $\phi$ designate the horizontal propagation direction, it follows that $u_{\|}^{\prime} \equiv \tilde{u_{\|}} \cos \left(\Phi_{u_{\|}}\right)$

$$
\begin{aligned}
& =u^{\prime} \cos (\phi)+v^{\prime} \sin (\phi) \\
& =\tilde{u} \cos \left(\Phi_{u}\right) \cos (\phi)+\tilde{v} \cos \left(\Phi_{v}\right) \sin (\phi)
\end{aligned}
$$

Let $A \equiv \tilde{u} \cos (\phi), B \equiv \tilde{v} \sin (\phi)$, and $\delta \phi \equiv \Phi_{v}-\Phi_{u}$, Eq. (A4) leads to

$$
\begin{aligned}
u_{\|}^{\prime} & =A \cos \left(\Phi_{u}\right)+B \cos \left(\Phi_{u}+\delta \phi\right) \\
& =A \cos \left(\Phi_{u}\right)+B \cos \left(\Phi_{u}\right) \cos (\delta \phi)-B \sin \left(\Phi_{u}\right) \sin (\delta \phi) \\
& =[A+B \cos (\delta \phi)] \cos \left(\Phi_{u}\right)-B \sin (\delta \phi) \sin \left(\Phi_{u}\right) \\
& =C_{1} \cos \left(\Phi_{u}\right)-C_{2} \sin \left(\Phi_{u}\right)
\end{aligned}
$$

where $C_{1} \equiv A+B \cos (\delta \phi)$ and $C_{2} \equiv B \sin (\delta \phi)$. Define

$\alpha \equiv \arctan \left(C_{2}, C_{1}\right)$

Equation (A5) can be rewritten as

$$
\begin{aligned}
u_{\|}^{\prime} & =\sqrt{C_{1}^{2}+C_{2}^{2}}\left[\cos (\alpha) \cos \left(\Phi_{u}\right)-\sin (\alpha) \sin \left(\Phi_{u}\right)\right] \\
& =\sqrt{C_{1}^{2}+C_{2}^{2}} \cos \left(\Phi_{u}+\alpha\right)
\end{aligned}
$$

which leads to

$\tilde{u_{\|}}=\sqrt{C_{1}^{2}+C_{2}^{2}}$

and

$\Phi_{u_{\|}}=\Phi_{u}+\alpha$

The relevant GW polarization relation in the Boussinesq approximation is

$\hat{T}^{\prime}=i \frac{N^{2}}{g \hat{\omega}} \frac{k_{h}}{m} u_{\|}^{\prime}$

Since $m<0$ and $k_{h}>0$ by convention, and $-i \cos (x)=\cos \left(x-\frac{\pi}{2}\right)$, thus

$\Phi_{T}=\Phi_{u_{\|}}-\frac{\pi}{2}$

Equations (A9) and (A11) lead to

$\Phi_{T}-\Phi_{u}-\alpha+\frac{\pi}{2}=0$

Note that $\phi$ can be either $\phi^{\prime}$ or $\phi^{\prime}+\pi$, depending on the phase relationship between winds and temperature, where $\phi^{\prime}$ is the orientation of the major axis of GW perturbation hodograph as derived using the Stokes parameter method. Equation (A12) is used to solve the $180^{\circ}$ ambiguity of $\phi$.

Acknowledgements. The authors are grateful to E. Jensen for insightful discussions on this paper. We appreciate the assistance of B. Gary in providing us the up-to-date information on the resolutions of the MTP data. We thank P. Minnis at the NASA Langley Research Center for providing assistance with the radar reflectivity images. Our thanks also go to the editor and two anonymous reviewers for valuable comments leading to clarification and improvement of the manuscript. The research by L. Wang and M. J. Alexander was supported by the National Science Foundation Physical \& Dynamical Meteorology Program 
grant ATM-0234230. Work performed by M. J. Mahoney at the Jet Propulsion Laboratory, California Institute of Technology, was done under contract with the National Aeronautics and Space Administration.

Edited by: K. Hamilton

\section{References}

Alexander, M. J., Beres, J. H., and Pfister, L.: Tropical stratospheric gravity wave activity and relationships to clouds, J. Geophys. Res., 105, 22 299-22 309, 2000.

Alexander, M. J. and Dunkerton, T. J.: A spectral parameterization of mean-flow forcing due to breaking gravity waves, J. Atmos. Sci., 56, 4167-4182, 1999.

Alexander, M. J. and Pfister, L.: Gravity wave momentum flux in the lower stratosphere over convection, Geophys. Res. Lett., 22, 2029-2032, 1995.

Alexander, M. J. and Vincent, R. A.: Gravity waves in the tropical lower stratosphere: A model study of seasonal and interannual variability, J. Geophys. Res., 105, 17 983-17 993, 2000.

Bacmeister, J. T., Eckermann, S. D., Newman, P. A., Lait, L., Chan, K. R., Loewenstein, M., Proffitt, M. H., and Gary, B. L.: Stratospheric horizontal wavenumber spectra of winds, potential temperature, and atmospheric tracers observed by high-altitude aircraft, J. Geophys. Res., 101, 9441-9470, 1996.

Bacmeister, J. T., Schoeberl, M. R., Lait, L. R., Newman, P. A., and Gary, B. L.: Small-scale waves encountered during AASE, Geophys. Res. Lett., 17, 349-352, 1990a.

Bacmeister, J. T., Schoeberl, M. R., Lait, L. R., Newman, P. A., and Gary, B. L.: ER-2 mountain wave encounter over Antarctica: Evidence for blocking, Geophys. Res. Lett., 17, 81-84, $1990 \mathrm{~b}$.

Beres, J., Alexander, M. J., and Holton, J. R.: A method of specifying the gravity wave spectrum above convection based on latent heating properties and background wind, J. Atmos. Sci., 61, 324$337,2004$.

Chun, H.-Y. and Baik, J.-J.: Momentum flux by thermally induced internal gravity waves and its approximation for large-scale models, J. Atmos. Sci., 55, 3299-3310, 1998.

Chun, H.-Y. and Baik, J.-J.: An updated parameterization of convectively forced gravity wave drag for use in large-scale models, J. Atmos. Sci., 59, 1006-1017, 2002.

Denning, R. F., Guidero, S. L., Parks, G. S., and Gary, B. L.: Instrument description of the airborne microwave temperature profiler, J. Geophys. Res., 94, 16757-16765, 1989.

Eckermann, S. D. and Vincent, R. A.: Falling sphere observations of anisotropic gravity wave motions in the upper stratosphere over Australia, Pure Appl. Geophys., 130, 509-532, 1989.

Eckermann, S. D.: Hodographic analysis of gravity waves: Relationsh ips among Stokes parameters, rotary spectra, and crossspectral methods, J. Geophys. Res., 101, 19 169-19 174, 1996.

Fritts, D. C. and Alexander, M. J.: Gravity wave dynamics and effects in the middle atmosphere, Rev. Geophys., 41(1), 1003, doi:10.1029/2001RG000106, 2003.

Gossard, E. E. and Hooke, W. H.: Waves in the Atmosphere, Elsevier Science, New York, 1975.

Haag, W. and Kärcher, B.: The impact of aerosols and gravity waves on cirrus clouds at midlatitudes, J. Geophys. Res., 109, D12202, doi:10.1029/2004JD004579, 2004.
Hertzog, A. and Vial, F.: A study of the dynamics of the equatorial lower stratosphere by use of ultra-long-duration balloons 2 . gravity waves, J. Geophys. Res., 106, 22 745-22 761, 2001.

Holton, J. R.: The role of gravity wave induced drag and diffusion in the momentum budget of the mesosphere, J. Atmos. Sci., 39, 791-799, 1982.

Isler, J. R., Taylor, M. J., and Fritts, D. C.: Observational evidence of wave ducting and evanescence in the atmosphere, J. Geophys. Res., 102, 26301-26313, 1997.

Jensen, E. J. and Pfister, L.: Transport and freeze-drying in the tropical tropopause layer, J. Geophys. Res., 109, D02207, doi:10.1029/2003JD004022, 2004.

Jensen, E. J., Pfister, L., Ackerman, A. S., and Tabazadeh, A.: A conceptual model of the dehydration of air due to freeze-drying by optically thin, laminar cirrus rising slowly across the tropical tropopause, J. Geophys. Res., 106, 17 273-17 252, 2001.

Jensen, E., Pfister, L., Bui, T. P., Weinheimer, A., Weinstock, E., Smith, J., Pittman, J., Baumgardner, D., Lawson, P., and McGill, M. J.: Formation of a tropopause cirrus layer observed over Florida during CRYSTAL-FACE, J. Geophys. Res., 110, D03208, doi:10.1029/2004JD004671, 2005.

Jensen, E. J., Starr, D., and Toon, O. B.: Mission investigates tropical cirrus clouds, EOS, 85, 45-50, 2004

Kraus, J. D.: Radio Astronomy, McGraw-Hill, New York, 1966.

Marks, C. J. and Eckermann, S. D.: A three-dimensional nonhydrostatic ray-tracing model for gravity waves: Formulation and preliminary results for the middle atmosphere, J. Atmos. Sci., 52, 1959-1984, 1995.

McFarlane, N. A.: The effect of Orographically excited gravity wave drag on the general circulation of the lower stratosphere and troposphere, J. Atmos. Sci., 44, 1775-1800, 1987.

Palmer, T. N., Shutts, G. J., and Swinbank, R.: Alleviation of a systematic westerly bias in general circulation and numerical weather prediction models through an orographic gravity wave drag parameterization, Quart. J. Roy. Meteorol. Soc., 112, 10011039, 1986.

Pfister, L., Chan, K. R., Bui, T. P., Bowen, S., Legg, M., Gary, B. L., Kelly, K., Proffitt, M., and Starr, W.: Gravity waves generated by a tropical cyclone during the step tropical field program: a case study, J. Geophys. Res., 98, 8611-8638, 1993.

Scott, S. G., Bui, T. P., Chan, K. R., and Bowen, S. W.: The meteorological measurement system on the NASA ER-2 aircraft, J. Atmos. Oceanic Technol., 7, 525-540, 1990.

Solomon, S.: Stratospheric ozone depletion: A review of concepts and history, Rev. Geophys., 37, 275-316, 1999.

Stockwell, R. G.: S-transform analysis of gravity wave activity from a small scale network of airglow imagers, Ph.D. thesis, The University of Western Ontario, 1999.

Stockwell, R. G. and Lowe, R. P.: Airglow imaging of gravity waves, 1 . Results from a small network of $\mathrm{OH}$ nightglow scanning imagers, J. Geophys. Res., 106, 17 185-17 203, 2001.

Stockwell, R. G., Mansinha, L., and Lowe, R. P.: Localisation of the complex spectrum: the S transform, J. Assoc. Expl. Geophys., XVII, 99-114, 1996.

Swenson, G. R., Alexander, M. J., and Haque, R.: Dispersion imposed limits on atmospheric gravity waves in the mesosphere: observations from oh airglow, Geophys. Res. Lett., 27, 875-878, 2000 . 
Vincent, R. A. and Alexander, M. J.: Gravity waves in the tropical lower stratosphere: An observational study of seasonal and interannual variability, J. Geophys. Res., 105, 17 971-17 982, 2000.

Vincent, R. A. and Fritts, D. C.: A climatology of gravity wave motions in the mesopause region at Adelaide, Australia, J. Atmos. Sci., 44, 748-760, 1987.

Wang, L.: Gravity wave analysis of four years of high vertical resolution U.S. radiosonde data, Ph.D. thesis, State University of New York at Stony Brook, 2003.
Wang, L., Geller, M. A., and Alexander, M. J.: Spatial and temporal variations of gravity wave parameters, Part I: Intrinsic frequency, wavelength, and vertical propagation direction, J. Atmos. Sci., 62, 125-142, 2005.

Wang, L., Fritts, D. C., Williams, B. P., Goldberg, R. A., Schmidlin, F. J., and Blum, U.: Gravity waves in the middle atmosphere during the MaCWAVE winter campaign: Evidence of mountain wave critical level encounters, Ann. Geophys., in press, 2006. 